\title{
Development of Heart Rate Variation Over the First 6 Months of Life in Normal Infants
}

\author{
VICKI L. SCHECHTMAN, RONALD M. HARPER, AND KAREN A. KLUGE \\ Department of Anatomy and Cell Biology and the Brain Research Institute, UCLA School of Medicine, \\ Los Angeles, California 90024
}

\begin{abstract}
Development of heart rate variation in three frequency ranges was examined during sleep-waking states in normal infants over the first 6 mo of life. Extent of all three types of heart rate variation decreased from 1 wk to 1 mo of age. Extent of respiratory sinus arrhythmia increased from 1 mo to 6 mo during all sleep-waking states, with the increase most pronounced during quiet sleep. Variation in two bands of lower frequencies showed increases in extent from 1 to 3 mo, then a slowing or reversal of the increase between 3 and 4 mo of age. During rapid eye movement sleep, the two types of lower frequency heart rate variation decreased in extent from 3 through 6 mo of age. These results suggest that alterations in autonomic control of heart rate occur at several time periods over the first 6 mo of life and that these alterations may have an effect only on particular types of heart rate variation and only during particular sleep-waking states. The diminution of all three types of heart rate variation at 1 mo may indicate a reduction in vagal tone at this age. (Pediatr Res 26: 343-346, 1989)
\end{abstract}

Abbreviations

AW, waking

QS, quiet sleep

REM, rapid eye movement

RSA, respiratory sinus arrhythmia

In healthy mammals, heart rate is not fixed, but undergoes moment-to-moment variation; the changes in heart rate may be rapid or prolonged. One prominent modulation of heart rate is RSA, which is characterized by increased heart rate during inspiration and slowing of the heart during expiration. RSA is most pronounced during QS (1) and is primarily mediated by the parasympathetic nervous system (2-4).

Two types of heart rate variation with periods longer than that of RSA have been identified in dogs and human adults. Oscillations with periods of approximately $10 \mathrm{~s}$ can be attributed to fluctuations in blood pressure (5) and are apparently mediated by the parasympathetic nervous system (3). Variation with periods of approximately $20 \mathrm{~s}$ is probably mediated by thermoregulatory mechanisms $(6,7)$ and is jointly controlled by both parasympathetic and $\beta$-sympathetic components of the autonomic nervous system (3).

Two major types of heart rate variation have been identified

Received November 2, 1988; accepted June 7, 1989.

Correspondence and reprint requests V. L. Schechtman, Ph.D., Brain Research Institute, Center for the Health Sciences, UCLA School of Medicine, Los Angeles, CA 90024-1761.

Supported by Grants HD-22695 and HD-22506 from the National Institute of Child Health and Human Development, U.S. Public Health Service. in infants: a high-frequency oscillation that is correlated with the respiratory cycle (RSA) (8-11) and lower frequency oscillations in a wide band of periods variously reported to range from 5 to 29 s (8-13). Radvanyi and Morel-Kahn (9) showed that the lower frequency variation is prominent in both QS and REM sleep before 37 wk gestational age. After $37 \mathrm{wk}$, RSA becomes the predominant type of heart rate variation in QS, and REM heart rate is characterized by RSA superimposed on lower frequency waves. Figure 1 shows heart rate variation during each sleepwaking state in one full-term infant at 2 mo postnatal age.

Using spectral analytic techniques, Harper et al. (14) showed that RSA during QS decreases over the 1st mo of life and that RSA during all sleep-waking states increases from 1 to 6 mo of age. The maturational trends in the lower frequency variation identified in infants have yet to be examined. Characterization of the development of this variation in early life could provide clues to the maturation of the underlying processes in infants. In this study we use a time-domain technique to assess the maturational changes in RSA and lower frequency heart rate variation during each sleep-waking state in normal infants from $1 \mathrm{wk}$ to 6 mo of age.

\section{MATERIALS AND METHODS}

Polygraphic recordings of EEG, ECG, electrooculogram, digastric electromyogram, and expired $\mathrm{CO}_{2}$ were obtained from 25 normal infants. Each infant was recorded on six occasions: at 1 wk of age and at 1, 2, 3, 4, and 6 mo of age. Each recording was begun at $1900 \mathrm{~h}$ and terminated at $0700 \mathrm{~h}$ the next morning. The ECG signal was fed into a Schmitt trigger generator that produced a pulse for each $\mathrm{R}$ wave. The resulting trigger event times were digitized along with the other signals.

Each 1-min epoch of data was classified as QS, REM sleep, AW, or indeterminate state by two trained observers using criteria similar to those suggested by Anders et al. (15) for state classification of newborns. Two of the 25 subjects were eliminated because of missing or distorted signals, leaving a total of 23 subjects, each with one recording at each of the six ages.

The intervals between successive $R$ waves of the ECG (R-R intervals) were calculated from the $R$ wave trigger times with an accuracy of $\pm 1 \mathrm{~ms}$, and breath-to-breath intervals were determined from the respiratory waveform. The median heart and respiratory rates for each 1-min epoch were determined from these R-R and breath-to-breath intervals. R-R intervals were subjected to an automated artifact detection and correction procedure (16), which identified those epochs containing considerable artifact as unsuitable for analysis. In addition, those epochs in which the median respiratory rate, calculated as the reciprocal of the median breath-to-breath interval, was artifactually high or low ( $>110$ breaths/min or $<15$ breaths/min) were eliminated from analysis. All $12 \mathrm{~h}$ of data, with the exception of those severely contaminated epochs, were used for analyses.

The corrected R-R interval records were subjected to a peak/ 

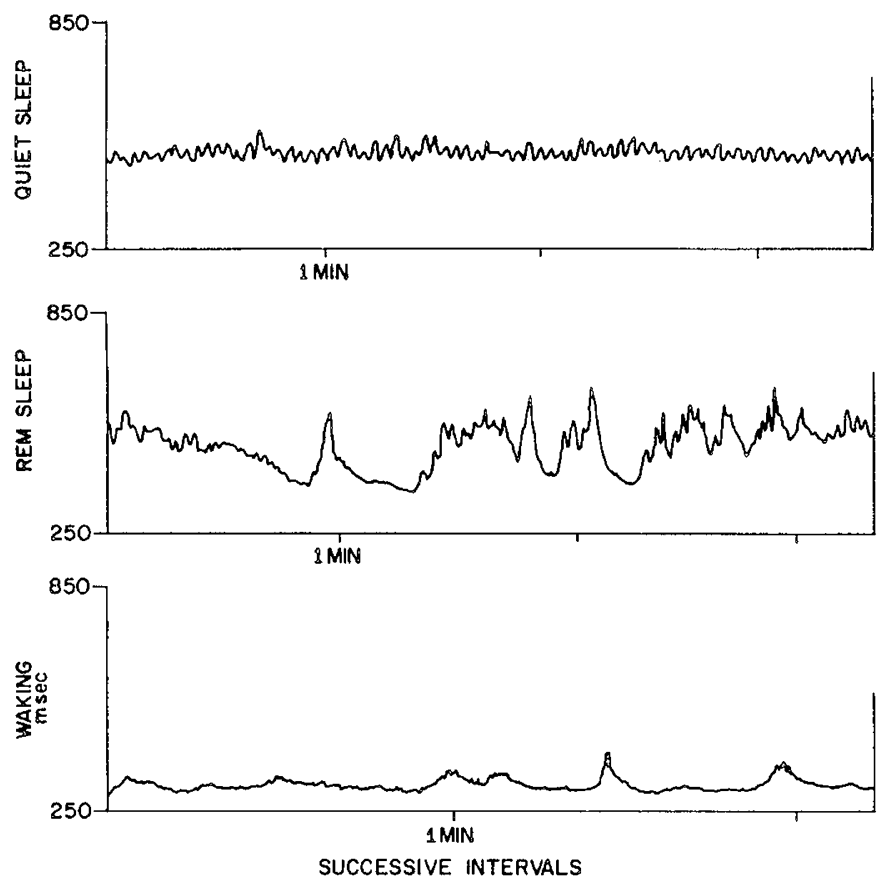

Fig. 1. Plots of cardiac R-R intervals from one 2-mo-old infant during different sleep-waking states. During quiet sleep, respiratory sinus arrhythmia is the predominant type of heart rate variation seen. REM sleep is characterized by respiratory sinus arrhythmia superimposed on large lower frequency changes in heart rate. During quiet waking, heart rate is relatively fixed with slight low frequency changes.

trough detection routine that calculated the period and extent (amplitude) of each variation in heart rate (16). This routine recognizes the highest frequency variation in an R-R interval record by identifying changes in the sign of the slope of the R-R interval curve as "peaks" and "troughs." Lower frequency variation is recognized as peaks and troughs in the curve formed by the peaks of the next higher frequency. The period of each variation is calculated by summing the R-R intervals between two peaks, and the extent of variation is represented by the peakto-trough difference in ms. Each variation in heart rate is thus described by two values representing its period and extent.

The median extent of all variation in each of three period ranges was determined for each epoch: 0.9 to $3.0 \mathrm{~s}$, predominantly respiratory-related heart rate variation (RSA), and 4.0 to $7.5 \mathrm{~s}$ and 12 to $30 \mathrm{~s}$, the two extremes of the lower frequency heart rate variation identified in infants. The lower frequency heart rate variation was thus divided into "midfrequency" and "low frequency" variation, rather than combining all variation with periods ranging from 4 to $30 \mathrm{~s}$. A broad frequency range including the bulk of respiratory activity in infants of this age (17) was chosen for assessment of RSA, rather than a narrower band at approximately the respiratory frequency, to ensure that all respiratory-related heart rate variation was assessed in each epoch despite differences in respiratory variability. For each type of heart rate variation, the median, rather than the mean, extent was used to prevent one or two aberrant changes in heart rate (e.g. a change caused by an apneic episode or other such event) from grossly affecting the measure of overall variability within the epoch.

For each recording, the median extent of each type of heart rate variation was determined for QS, REM sleep, and AW. The use of the median rather than the mean provided a more stable measure of extent, in light of considerable variability in minuteby-minute estimates. For each type of heart rate variation, a twoway analysis of variance [BMDP2V (18)] was performed to assess differences in heart rate variation over the six ages and the three sleep-waking states.

\section{RESULTS}

$R S A$. Figure 2 shows the mean extent of RSA during QS, REM sleep, and AW at each age. RSA was diminished at 1 mo of age during all three sleep-waking states. During the first 2 mo of life, extent of RSA was similar in QS and REM sleep. After 2 mo of age, however, extent of RSA showed much more rapid maturational increases during QS than during REM sleep. Extent of RSA was lower during AW than during either sleep state at all ages. Analysis showed a significant age $\times$ state interaction $(p$ $<0.0001)$, as well as significant simple main effects for both variables ( $p<0.0001$ for both age and state).

Midfrequency heart rate variation. Mean extent of midfrequency heart rate variation during each sleep-waking state is shown in Figure 3. Extent of midfrequency heart rate variation was greatest during REM sleep at all ages. Midfrequency heart rate variation was diminished at 1 mo of age in all sleep-waking states. During QS, extent of midfrequency heart rate variation increased from 1 through 6 mo of age. During REM sleep, extent of this type of variation increased from 1 to $3 \mathrm{mo}$ of age, and then decreased through 6 mo. During AW, there was a slight decrease in extent of midfrequency heart rate variation from 3 to 4 mo of age, but the increasing trend resumed after 4 mo of age. Analysis showed a significant state $\times$ age interaction $(p<$ $0.0001)$ and simple main effects for both state $(p<0.0001)$ and age $(p<0.0001)$.

Low frequency heart rate variation. Figure 4 shows mean extent of low frequency heart rate variation during QS, REM sleep, and $\mathrm{AW}$ over the first 6 mo of life. Extent of low frequency heart rate variation also decreased over the 1 st mo of life during all sleep-waking states. In all three states, the developmental patterns were similar to those of midfrequency heart rate variation. Again, analysis showed a significant state $\times$ age interaction $(p<0.0004)$

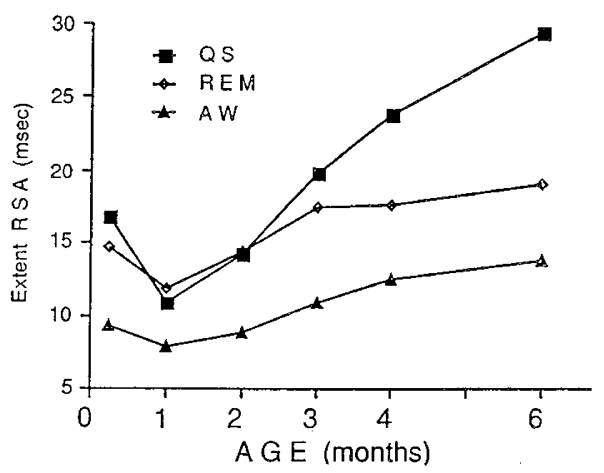

Fig. 2. Mean extent of RSA during QS, REM sleep, and AW in 23 normal infants over the first 6 mo of life. Extent of RSA decreases over the 1 st mo of life during all states.

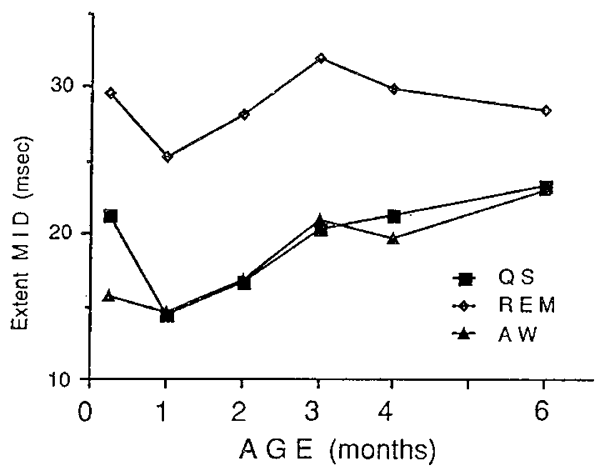

Fig. 3. Mean extent of midfrequency heart rate variation (MID) during each state in 23 normal infants over the first 6 mo of life. Midfrequency heart rate variation is diminished at 1 mo during all states, and declines after 3 mo during REM sleep. 
and simple main effects of both state $(p<0.0001)$ and age $(p<$ 0.0001 ).

The mean and SE for each type of heart rate variation in each sleep state and each age group are shown in Table 1 . Notice that RSA is most prominent in QS in the older infants and that both mid- and low frequency heart rate variation are most prominent during REM sleep in all age groups.

\section{DISCUSSION}

These results confirm previous findings that, during QS, extent of RSA decreases over the 1st mo of life and then gradually increases (14). Our results differ from the previous findings in several ways. We found RSA to be diminished at 1 mo of age during all sleep-waking states, whereas the spectral study showed a diminution only during QS. We also found extent of RSA to be similar in QS and REM sleep during the first 2 mo of life, whereas spectral analysis showed RSA to be more prominent during QS at all ages up to $6 \mathrm{mo}$. In addition, peak/trough techniques showed extent of RSA during QS to increase steeply through the 6th mo of life, whereas spectral analysis showed only minor changes after 3 mo of age.

All these differences between the two studies may result from differences in the manner in which RSA is quantified by the two methods. Using spectral analysis, heart rate variation is assessed

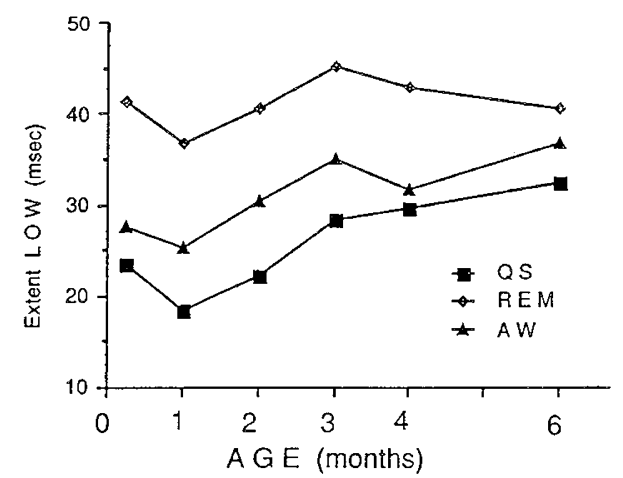

Fig. 4. Mean extent of low frequency heart rate variation ( $L O W)$ during each state in 23 normal infants over the first 6 mo of life. In each state, the developmental pattern of low frequency heart rate variation is similar to that of midfrequency heart rate variation (Fig. 3).

Table 1. Means and SE for extent of three types of heart rate variation during each sleep state over first 6 mo of life

\begin{tabular}{lcccr}
\hline & Age & QS & REM & \multicolumn{1}{c}{ AW } \\
\hline RSA & $1 \mathrm{wk}$ & $16.8(1.6)$ & $14.7(1.3)$ & $9.3(0.8)$ \\
& $1 \mathrm{mo}$ & $10.8(0.6)$ & $11.8(0.6)$ & $7.9(0.4)$ \\
& $2 \mathrm{mo}$ & $14.3(1.5)$ & $14.3(1.2)$ & $8.9(0.7)$ \\
& $3 \mathrm{mo}$ & $19.7(1.7)$ & $17.5(1.4)$ & $10.9(1.0)$ \\
& $4 \mathrm{mo}$ & $23.8(2.2)$ & $17.7(1.5)$ & $12.5(1.6)$ \\
& $6 \mathrm{mo}$ & $29.3(3.1)$ & $19.0(2.2)$ & $13.7(1.5)$ \\
Midfrequency & $1 \mathrm{wk}$ & $21.3(1.9)$ & $29.6(2.0)$ & $15.8(1.4)$ \\
heart rate varia- & $1 \mathrm{mo}$ & $14.4(0.9)$ & $25.2(1.1)$ & $14.6(1.0)$ \\
tion & $2 \mathrm{mo}$ & $16.7(1.2)$ & $28.2(1.5)$ & $16.8(1.4)$ \\
& $3 \mathrm{mo}$ & $20.3(1.3)$ & $32.0(1.5)$ & $21.0(1.4)$ \\
& $4 \mathrm{mo}$ & $21.3(1.3)$ & $29.9(1.6)$ & $19.7(2.0)$ \\
& $6 \mathrm{mo}$ & $23.4(1.6)$ & $28.5(1.6)$ & $23.1(1.9)$ \\
Low frequency & & & & \\
heart rate varia- & $1 \mathrm{wk}$ & $23.5(2.0)$ & $41.4(2.8)$ & $27.6(2.2)$ \\
tion & $2 \mathrm{mo}$ & $18.3(1.3)$ & $36.7(1.9)$ & $25.3(1.6)$ \\
& $2 \mathrm{mo}$ & $22.2(1.8)$ & $40.7(2.4)$ & $30.4(2.4)$ \\
& $3 \mathrm{mo}$ & $28.3(1.8)$ & $45.2(2.2)$ & $34.9(2.9)$ \\
& $4 \mathrm{mo}$ & $29.7(1.5)$ & $42.8(2.4)$ & $31.6(2.0)$ \\
& $6 \mathrm{mo}$ & $32.3(1.7)$ & $40.6(2.1)$ & $36.7(2.5)$ \\
\hline
\end{tabular}

by fitting various sine waves within the frequency range of interest to the signal, and measuring the goodness of fit of the signal to the sine waves; thus, spectral techniques are most appropriately applied to sinusoidal, or near-sinusoidal, variation. However, peak/trough techniques are less sensitive to the shape of variation, and assess equally all variation with a given peakto-peak period, regardless of asymmetry or other nonsinusoidal features. Thus, differences in the sinusoidal nature of variation might account for differences in results obtained by spectral versus peak/trough methods. The use of a wider (and standardized) bandwidth for RSA in our study might also account for the discrepancy between these findings and the findings obtained using spectral analysis. The use of this wider bandwidth has the advantage of measuring all respiratory-related heart rate variation despite wide differences in respiratory variability, but the disadvantage of possible inclusion of some heart rate variation from nonrespiratory sources. Finally, the extent of lower frequency variation may affect the measurement of extent of higher frequency variation using the peak/trough method, whereas this bias may be avoided in spectral analysis with appropriate prewhitening techniques as were used by Harper et al. (14). This effect is minimized, however, by use of the median rather than the mean as the measure of central tendency in the peak/trough method, and visual examination of the data did not show contamination from lower frequency variation to significantly affect measurements.

Our finding that extent of RSA is similar in QS and REM sleep during the first 2 mo of life may reflect immaturity of sleep state differentiation during this period. The finding that, during QS, extent of RSA increases steadily from 1 through 6 mo of age, whereas during REM sleep, extent of this variation plateaus after $3 \mathrm{mo}$, may anticipate the greater extent of RSA during QS relative to REM sleep previously demonstrated in adults (1).

We have found that extent of all types of heart rate variation decreases over the 1 st mo of life, and that these decreases are apparent during all sleep-waking states, suggesting that the previous finding of diminished RSA at 1 mo of age (14) may not be a result of a specific change in the cardiac-respiratory interaction, but possibly the result of a general decrease in parasympathetic control of the heart during this period. Such a decrease in cardiac parasympathetic control would also account for the increased heart rate observed in infants at this age (19). Although an increase in sympathetic outflow might also account for increased heart rate, sympathetic activity has little effect on RSA (2-4) or the 10 -s blood pressure oscillation (3) in adults; thus, it is unlikely that an increase in sympathetic activity could account for the decreases in heart rate variation.

Mid- and low frequency heart rate variation followed very similar maturational patterns. Both types of variation showed decreased extent over the 1st mo of life, followed by steep increases over the next 2 mo in all sleep states. From 3 to 6 mo of age, the developmental pattern was different for each sleepwaking state, but for each state, mid-and low frequency variation showed similar patterns. During all states, the steep increases in both types of variation ended at 3 mo of age, but only during REM sleep did extent of variation begin a decline at this age that lasted through 6 mo of age. The similarity of the developmental patterns of mid- and low frequency heart rate variation suggests that, despite the wide range of frequencies, variation with periods ranging from 4 to $30 \mathrm{~s}$ may be controlled by similar mechanisms.

Blood pressure and thermoregulatory influences have been shown to cause heart rate variation in adults (5-7) and must, therefore, be considered as possible sources of the mid- and low frequency variation observed in infants. Several other factors may also contribute to heart rate variation in the mid- and low frequency bands. Movement is associated with variation in heart rate in the fetus (20-23) and the infant $(22,24)$, as well as in adults (25). Respiratory pauses, which are common in infants (26), also cause changes in heart rate (27), as do sucking and crying behaviors. Developmental changes in these factors may 
contribute to the maturational patterns of mid- and low frequency heart rate variation.

It is apparent from these results that maturation of the mechanisms contributing to heart rate variation does not occur linearly over the first 6 mo of life. Between $1 \mathrm{wk}$ and 1 mo of age, some factor (possibly a decrease in parasympathetic tone to the heart) causes a sudden diminution of all types of heart rate variation. Extent of all types of heart rate variation then increases from age 1 to 3 mo. Between 3 and 4 mo of age, some factor slows or reverses the increasing trends, and during REM sleep, the decrease in lower frequency heart rate variation continues into the 6 th mo of life. These developmental changes in heart rate variation provide us with insights into the development of autonomic control systems in infants.

\section{REFERENCES}

1. Trelease RB, Harper RM, Sieck GC 1981 Respiratory-related heart rate variation during sleep and waking states in cats. Exp Neurol 72:195-203

2. Katona PG, Jih F 1975 Respiratory sinus arrhythmia: noninvasive measure of parasympathetic cardiac control. J Appl Physiol 39:801-805

3. Akselrod S, Gordon D, Ubel FA, Shannon DC, Barger AC, Cohen RJ 1981 Power spectrum analysis of heart rate fluctuation: a quantitative probe of beat-to-beat cardiovascular control. Science 213:220-222

4. Coker R, Koziell A, Oliver C, Smith SE 1984 Does the sympathetic nervous system influence sinus arrhythmia in man? Evidence from combined autonomic blockade. J Physiol 356:459-464

5. de Boer RW, Karemaker JM, Strackee J 1985 Relationships between shortterm blood-pressure fluctuations and heart-rate variability in resting subjects. I: a spectral analysis approach. Med Biol Eng Comp 23:352-358

6. Kitney RI 1975 An analysis of the nonlinear behaviour of the human thermal vasomotor control system. J Theor Biol 52:231-248

7. Kitney RI, Rompelman O 1977 Analysis of the interaction of the human blood pressure and thermal control systems. In: Perkins WJ (ed) Biomedical Computing. University Park Press, Baltimore, pp 49-54

8. Tarlo PA, Valimaki I, Rautaharju PM 1971 Quantitative computer analysis of cardiac and respiratory activity in newborn infants. J Appl Physiol 31:7075

9. Radvanyi MF, Morel-Kahn F 1976 Sleep and heart rate variations in premature and full term babies. Neuropadiatrie 7:302-312

10. Miyazaki S, Watanabe K, Hara K 1979 Heart rate variability in full-term normal and abnormal newborn infants during sleep. Brain Dev 1:57-60

11. Giddens DP, Kitney RI 1985 Neonatal heart rate variability and its relation to respiration. J Theor Biol 113:759-780

12. Yeh S-Y, Forsythe A, Hon EH 1973 Quantification of fetal heart beat-to-beat interval differences. Obstet Gynecol 41:355-363

13. Kitney RI 1984 New findings in the analysis of heart rate variability in infants. Automedica 5:289-310

14. Harper RM, Walter DO, Leake B, Hoffman HJ, Sieck GC, Sterman MB, Hoppenbrouwers T, Hodgman J 1978 Development of sinus arrhythmia during sleeping and waking states in normal infants. Sleep 1:33-48

15. Anders TF, Emde R, Parmelee A 1971 A Manual of Standardized Terminology, Techniques and Criteria for Scoring of Sleep and Wakefulness in Newborn Infants. UCLA Brain Information Service/BRI Publication Office, Los Angeles

16. Schechtman VL, Kluge KA, Harper RM 1988 Time-domain system for assessing variation in heart rate. Med Biol Eng Comp 26:367-373

17. Hoppenbrouwers $T$, Harper RM, Hodgman JE, Sterman MB, McGinty DJ 1978 Polygraphic studies of normal infants during the first six months of life. II. Respiratory rate and variability as a function of state. Pediatr Res 12:120-125

18. Dixon WJ, Brown MB, Engelman L, Frane JW, Hill MA, Jennrich RI, Toporek JD 1985 BMDP Statistical Software. University of California Press, Los Angeles, pp 359-387

19. Harper RM, Leake B, Hodgman JE, Hoppenbrouwers T 1982 Developmental patterns of heart rate and heart rate variability during sleep and waking in normal infants and infants at risk for the sudden infant death syndrome. Sleep 5:28-38

20. Junge HD 1979 Behavioral states and state related heart rate and motor activity patterns in the newborn infant and the fetus ante partum-a comparative study. I. Technique, illustration of recordings, and general results. J Perinat Med 7:85-107

21. Junge HD 1979 Behavioral states and state related heart rate and motor activity in the newborn infant and the fetus ante partum - a comparative study. II. Computer analysis of state related heart rate baseline and macrofluctuation patterns. J Perinatal Med 7:134-148

22. Junge HD 1980 Behavioral states and state-related heart rate and motor activity patterns in the newborn infant and the fetus ante partum-a comparative study. III. Analysis of sleep state-related motor activity patterns. Eur J Obstet Gynecol Reprod Biol 10:239-246

23. Timor-Tritsch IE, Dierker LJ, Zador I, Hertz RH, Rosen MG 1978 Fetal movements associated with fetal heart rate accelerations and decelerations. Am J Obstet Gynecol 131:276-280

24. Erkinjuntti M, Kero P 1985 Heart rate response related to body movements in healthy and neurologically damaged infants during sleep. Early Hum Dev $12: 31-37$

25. Alihanka $\mathbf{J} 1982$ Sleep movements and associated autonomic nervous activities in young male adults. Acta Physiol Scand [Suppl] 511:1-85

26. Richards JM, Alexander JR, Shinebourne EA, de Swiet M, Wilson AJ, Southall DP 1984 Sequential 22-hour profiles of breathing patterns and heart rate in 110 full-term infants during their first 6 months of life. Pediatrics 74:763777

27. Burack B 1984 The hypersomnia-sleep apnea syndrome: its recognition in clinical cardiology. Am Heart J 107:543-548 